\title{
Proton beam therapy for locally advanced and unresectable (T4bN0M0) squamous cell carcinoma of the ethmoid sinus: A report of seven cases and a literature review
}

\author{
TAKASHI SAITO, HITOSHI ISHIKAWA, KAYOKO OHNISHI, TERUHITO AIHARA, MASASHI MIZUMOTO, \\ NOBUYOSHI FUKUMITSU, KAORI SUGAWARA, TOSHIYUKI OKUMURA and HIDEYUKI SAKURAI \\ Department of Radiation Oncology, University of Tsukuba, Faculty of Medicine, Tsukuba, Ibaraki 305-8575, Japan
}

Received August 6, 2014; Accepted April 14, 2015

DOI: $10.3892 / \mathrm{ol} .2015 .3214$

\begin{abstract}
The present study reports treatment outcomes of locally advanced and unresectable squamous cell carcinoma of the ethmoid sinus (SCC-ES) following proton beam therapy (PBT). Between January 1997 and December 2012, 7 patients (median age, 63 years) with SCC-ES underwent definitive PBT. All tumors were categorized as T4bNOM0 (2009 UICC tumor-node-metastasis classification) and were treated using conventional fractionation at a median total dose of 72 Gy equivalents (GyE; range, 70.4-76 GyE). Imaging diagnosis for the initial treatment effect within 3 months of PBT revealed that a complete response (CR) was achieved in 2 patients and a partial response (PR) in 5 patients. The overall median survival time of the patients was 43 months (range, 12-62 months), and 4 patients survived for $\geq 3$ years. No recurrence was observed in the 2 patients who exhibited an initial CR treatment effect; however, locoregional recurrences occurred in $4 / 5$ patients who exhibited a PR. No grade 3 or severe acute toxicities were observed, but the late toxicities of grade 3 contralateral optic nerve damage and cataracts developed in 1 and 2 patients, respectively. Based on the findings of the present study, intensification of the local treatment effect may be important for yielding favorable treatment outcomes, since no distant metastasis was observed. PBT is therefore a potentially useful treatment tool for unresectable SCC-ES.
\end{abstract}

\section{Introduction}

Malignancies of the paranasal cavity are rare, representing $3 \%$ of all head and neck cancers and $\leq 1 \%$ of total malignancies; the

Correspondence to: Dr Hitoshi Ishikawa, Department of Radiation Oncology, University of Tsukuba, Faculty of Medicine, 1-1-1 Tennodai, Tsukuba, Ibaraki 305-8575, Japan

E-mail: hishikawa@pmrc.tsukuba.ac.jp

Key words: ethmoid sinus carcinoma, local control, proton beam therapy, radiation therapy, toxicity combination of surgery, chemotherapy and radiation therapy (RT) has been widely used in patients with the disease (1-3). Among paranasal cavity malignancies, cancer of the ethmoid sinus complex is even rarer, and surgical approaches are usually complicated by a lack of satisfactory surgical clearance and the risk of serious dysfunction of the surrounding normal tissues, including the brain stem and optic nerve (4). Consequently, definitive RT for patients with unresectable squamous cell carcinoma of the ethmoid sinus (SCC-ES) has been performed, but it can be difficult to deliver a curative irradiation dose to the tumor without severe complications, such as visual loss and brain necrosis, among others (5). Thus, there is little information on the efficacy of RT for SCC-ES at present.

Proton beam therapy (PBT) offers advantageous physical properties to RT for a variety of cancers. Since proton beams exhibit a spread-out Bragg peak and achieve an improved dose distribution of the target volume using specified beam modulations compared with photon beams (6-10), PBT delivers a large irradiation dose to the tumor using limited numbers of portals while sparing the surrounding normal tissues. Hence, the technique may yield improved disease control with minimum morbidity compared with previous conventional RT (11). A number of previous studies have reported favorable outcomes for patients with head and neck cancers treated with PBT (8-10), but SCC-ES has not been an area of focus, as there is a limited number of patients with this disease. Therefore, the efficacy of PBT in addition to RT using photon beams in the management of SCC-ES remains unknown.

The present study reports the clinical outcomes of patients with SCC-ES who were treated with PBT for the first time, and reviews the literature regarding the role of RT in the management of SCC-ES. Furthermore, three-dimensional conformal RT (3D-CRT) and intensity-modulated RT (IMRT) treatment planning using the same CT images was performed for the most recently treated SCC-ES case in this series. The dose-volume histograms of tumor and normal tissues, including the optic nerve, chiasm, brain and brain stem, from 3D-CRT and IMRT were then compared with those from PBT in order to assess the physical advantages of using proton beams for the treatment of SCC-ES. 
Table I. Characteristics of the 7 patients.

\begin{tabular}{lccccc}
\hline No. & Age & Gender & Radiation therapy & Dose fractionation & $\begin{array}{c}\text { Chemotherapy } \\
\text { (cycles, } \mathrm{n} \text { ) }\end{array}$ \\
\hline 1 & 79 & Female & X-ray and PBT & $70.4 \mathrm{GyE} / 38 \mathrm{~F}$ & No \\
2 & 58 & Male & X-ray and PBT & $70.4 \mathrm{GyE} / 38 \mathrm{~F}$ & No \\
3 & 46 & Male & PBT & $72.0 \mathrm{GyE} / 36 \mathrm{~F}$ & No \\
4 & 53 & Female & PBT & $72.0 \mathrm{GyE} / 36 \mathrm{~F}$ & No \\
5 & 63 & Female & PBT & $74.0 \mathrm{GyE} / 37 \mathrm{~F}$ & Induction FP (2) \\
6 & 71 & Male & PBT & $74.0 \mathrm{GyE} / 37 \mathrm{~F}$ & Concurrent FP(2) \\
7 & 64 & Female & PBT & $76.0 \mathrm{GyE} / 38 \mathrm{~F}$ & \\
\hline
\end{tabular}

PBT, proton beam therapy; FP, 5-fluorouracil and cisplatin; GyE, Gy equivalents.

\section{Case report}

Patients. A total of 7 patients with SCC originating from the ethmoid sinus were reviewed retrospectively in this study. The patients were treated with definitive PBT at the University of Tsukuba Hospital (Ibaraki, Japan) between January 1997 and December 2012. Patients with recurrent disease or tumors originating from other regions were excluded. Details of the patients' characteristics are presented in Table I. The median age of the patients was 63 years, ranging from 46-79 years. Written informed consent was obtained from all subjects prior to the initiation of treatment.

Pretreatment evaluation was performed by physical examination, nasopharyngeal endoscopy, computed tomography (CT) and magnetic resonance imaging (MRI). All tumors evaluated in the present study were categorized as T4bN0M0 based on sections of the nasal cavity and paranasal sinuses, according to the 7 th edition of the tumor-node-metastasis classification of the International Union Against Cancer (2009) (12).

Proton beam therapy. The RT treatment policy for the present study has been described previously (13). PBT planning was performed on a 3D CT planning system with a $5-\mathrm{mm}$ slice thickness. Patients were immobilized in the supine position using a thermoplastic mask. In principle, the clinical target volume (CTV) included the gross tumor volume and bilateral ethmoid sinuses, and the initial planning target volume was determined by adding margins of $8-10 \mathrm{~mm}$ to the CTV. To spare the surrounding normal tissues and to adapt the planning target volume to the tumor volume reduction, treatment plans were commonly changed twice to the total doses of 30-40 Gy equivalents (GyE) and 60 GyE (Fig. 1). Dose constraints for the organs at risk were as follows: Optic nerve of the healthy side/chiasm, $50 \mathrm{GyE}$; optic lens, $10 \mathrm{GyE}$; and brainstem surface, $50 \mathrm{GyE}$. However, in certain cases where the tumor was adjacent to these organs, it was not possible to follow these constraints. A median total total dose of $72 \mathrm{GyE}$ (range, 70.4-76 GyE) was delivered at a fractional dose of 1.8-2.0 GyE. Patient 6 underwent two 28-day cycles of induction chemotherapy with 5-fluorouracil (5-FU; $800 \mathrm{mg} / \mathrm{m}^{2}$, days, 1-5) and cisplatin (CDDP; $60 \mathrm{mg} / \mathrm{m}^{2}$, day 1) prior to PBT treatment. Patient 7 underwent two 28-day cycles of chemotherapy with 5-FU
(700 mg/m², days $1-5)$ and cisplatin (70 mg/m², day 6), which was administered concurrently with PBT (Table I).

Follow-up, treatment efficacy and toxicity evaluation. The last follow-up was performed in February 2014, and the median follow-up time was 3.6 years. The follow-up included a physical examination and nasopharyngeal endoscopy, which was performed at 1-2 month intervals during the first year following completion of PBT and at 2-3 month intervals thereafter.

The initial treatment effect was evaluated using the RECIST 1.0 criteria (14) based on diagnostic imaging by $\mathrm{CT}$ and/or MRI at 2-3 months following the completion of PBT. The diagnostic imaging was repeated routinely every 6 months thereafter. The toxicities were graded according to the Common Terminology Criteria for Adverse Events version 4.0 (15).

\section{Results}

Efficacy and failure pattern. The details of the treatment results are presented in Table II and Fig. 2. Evaluation of the initial treatment response demonstrated a complete response (CR) in 2 patients $(29 \%)$ and a partial response (PR) in the remaining 5 patients. The median survival time of all patients was 43 months (range, 12-62 months).

Recurrences were observed in $4 / 5(80 \%)$ patients in the PR group according to the initial tumor response 1-22 months after the completion of PBT. The initial recurrence sites were the primary tumor lesion in 4 patients and the regional lymph nodes in 1 patient, respectively, but no distant metastases were detected. In addition, there were no recurrences in the CR group.

Toxicity. Prior to the start of PBT, 3 patients presented with visual impairment, and another patient presented with ipsilateral visual loss due to the disease. These conditions were therefore not included among the toxicities induced by the treatment in the present study. There was no grade 3 or severe acute toxicity. Regarding late toxicity, grade 3 contralateral optic nerve damage and cataracts were observed in 2 and 1 patient, respectively, grade 1 brain necrosis was observed in 1 patient, and 2 brain necrosis was observed in 1 patient. In 
Table II. Treatment outcome and observed toxicity in the 7 patients.

\begin{tabular}{lccccc}
\hline No. & Initial effect & Recurrence (time) & Status & Survival time, months & Late toxicity (grade) \\
\hline 1 & CR & None & NED & 62 & Cataracts (3), contralateral optic nerve damage (3) \\
2 & PR & Local (5 months) & DWD & 12 & None \\
3 & PR & None & DOD & 53 & Brain necrosis (1) \\
4 & PR & Local (22 months) & DWD & 43 & Cataracts (3) \\
5 & PR & Local+LN (1 month) & DWD & 12 & None \\
6 & PR & Local (2 months) & DWD & 47 & None \\
7 & CR & None & DOD & 20 & Brain necrosis (2)
\end{tabular}

CR, complete response; PR, partial response; NED, no evidence of disease; DWD, dead with disease; DOD, died of other disease; LN, lymph nodes.

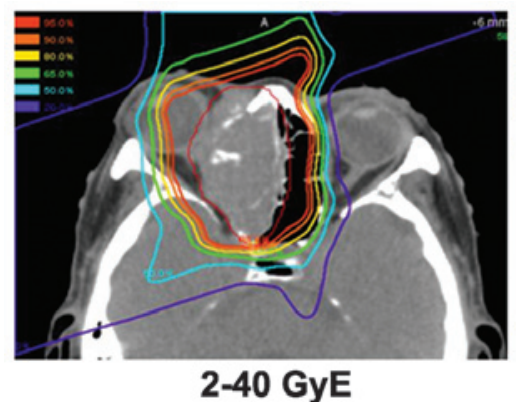

2-40 GyE

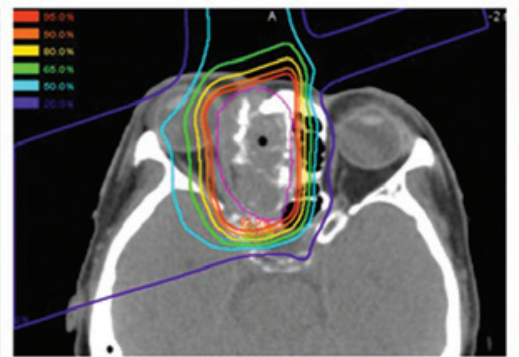

42-60 GyE

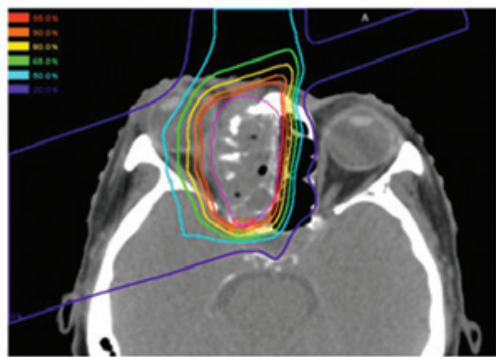

62-76 GyE

Figure 1. Representative dose distribution of proton therapy for ethomoid sinus squamous cell carcinoma (target, magenta. Isodose lines: $95 \%$, red; $90 \%$, orange; $80 \%$, yellow; $60 \%$, green; $50 \%$, blue; $20 \%$, purple).
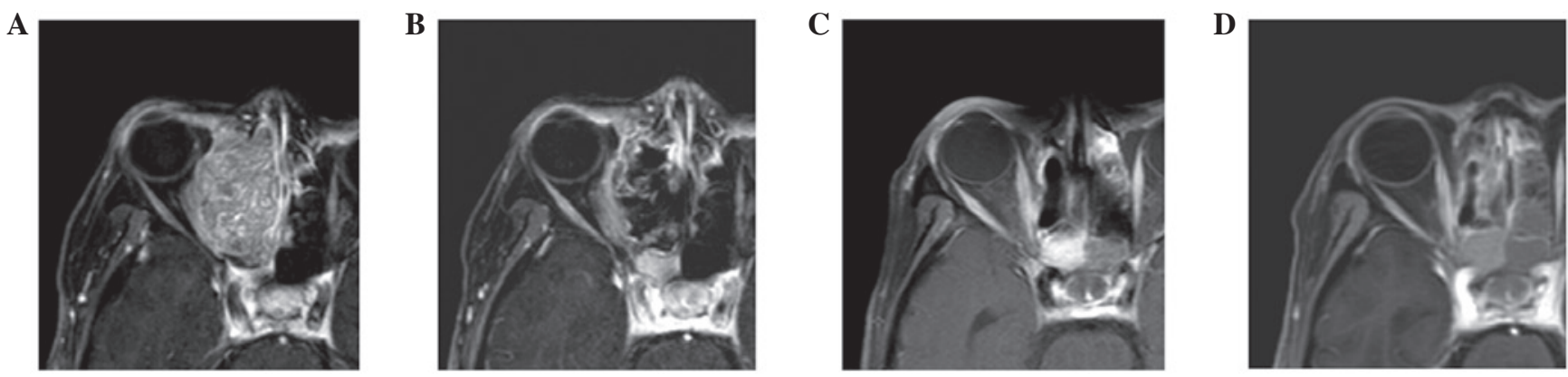

Figure 2. Patient 7. Change in the tumor prior to and following proton beam therapy based on representative magnetic resonance images. (A) Prior to treatment, (B) immediately after completion of treatment, (C) 9 months after treatment and (D) 16 months after treatment; this tumor completely regressed 9 months after proton beam therapy. (D) Inflammatory changes were observed, but there was no tumor recurrence in the 16 months following the treatment.
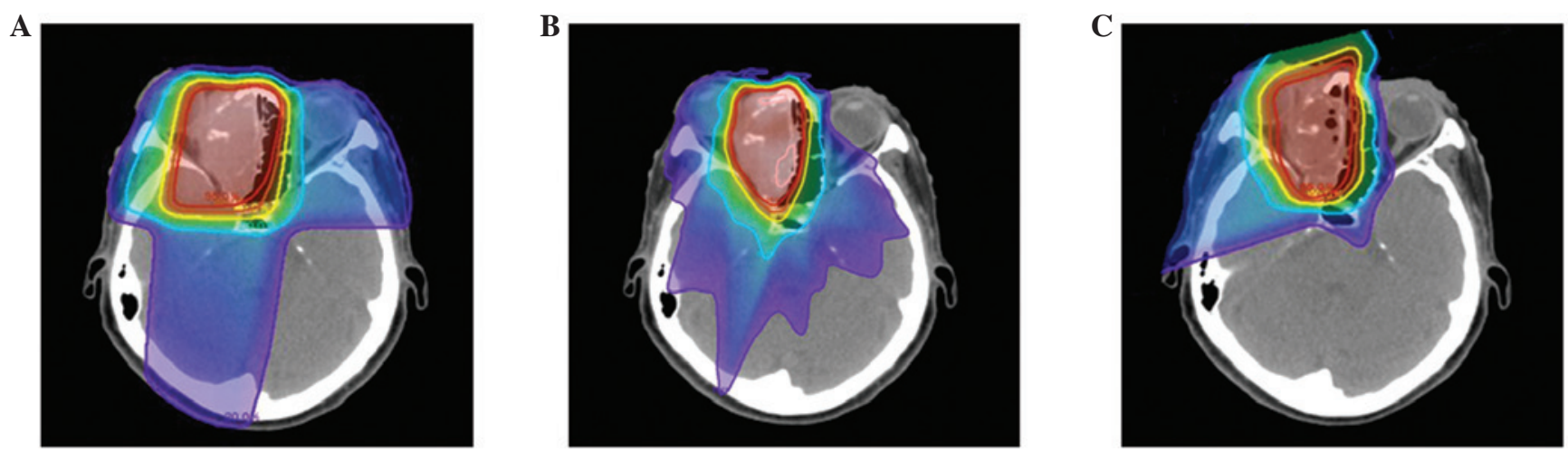

Figure 3. Patient 7. Comparison of dose distributions according to the treatment method. (A) Three-dimensional conformal radiation therapy, (B) intensity-modulated radiation therapy and (C) proton beam therapy. 
A

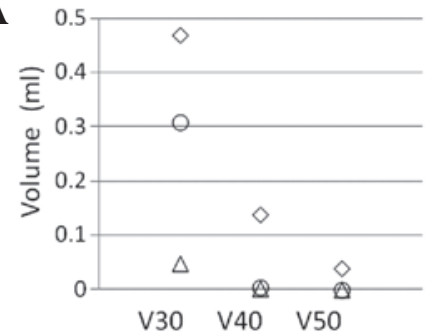

C

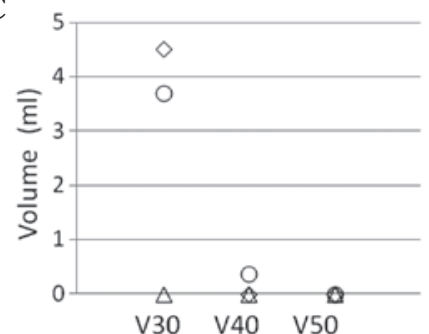

B $\diamond 3 \mathrm{D}$-CRT OIMRT $\triangle$ proton

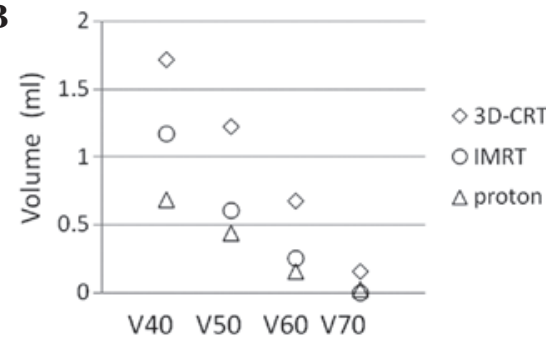

D

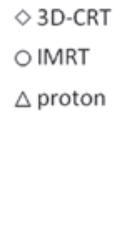

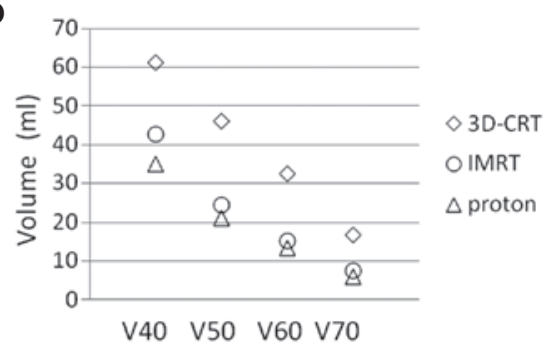

Figure 4. Patient 7. Comparison of dose-volume histograms of risk organs according to treatment methods: (A) Contralateral optic nerve, (B) chiasm, (C) brain stem and (D) brain. V30 indicates the volume (ml) of the organs at risk irradiated at $30 \mathrm{~Gy}$.

the brain necrosis case, the irradiation field included the brain tissues surrounding the tumor, and a total dose of 70 GyE was delivered, even though the shrinking field technique used for boost therapy was adopted. For all 3 cases with visual impairment prior to the start of PBT, their symptoms did not improve following treatment.

\section{Discussion}

The reported treatment outcomes, including quality of life, have been disappointing for patients with SCC-ES, even if they were candidates for radical surgery. Despite RT being an alternative curative treatment method, it is difficult to administer a high irradiation dose to the tumor without severe complications $(16,17)$. Consequently, comparing the superiority of treatment modalities for SCC-ES, such as surgery and RT combined with or without chemotherapy, is made difficult by the lack of availability of information concerning SCC-ES treatment outcomes, mainly due to the rarity of this disease.

Waldron et al (5) reported that the 5-year survival rates and the local tumor control with RT alone were affected by the tumor T-stage and histological type. In particular, local recurrences were observed in $8 / 11(72 \%)$ patients with T4 SCC-ES, and almost all recurrences developed within a year of treatment. In addition, the median overall survival time for the 11 patients was $\leq 12$ months. However, in the present study, local tumor control was achieved in 3/7 (43\%) patients with T4 SCC-ES, and the median survival time was 43 months. It appears that the higher local control rate in the present study was mainly due to the high irradiation doses administered to the primary tumor, although the number of subjects was small. Since a previous study demonstrated that a total dose of $\geq 65 \mathrm{GyE}$ is a preferable factor for improvement of local tumor control and overall survival (16), and since all recurrences in the present study actually developed at the primary tumor site, intensification of the local treatment may be important to improve the outcomes of SCC-ES.
Nevertheless, there are certain issues with administering high irradiation doses to the SCC-ES primary tumor. In particular, there are a number of vulnerable organs in this region, including the brain, brain stem and optic nerve, and it is potentially difficult to deliver $\geq 65$ Gy to the tumor using conventional RT methods without inducing severe late complications, such as optic nerve damage and brain necrosis $(16,17)$. To reduce the irradiation dose to organs at risk, intensity-modulated radiation therapy (IMRT) and PBT are often used, and Mock et al (18) reported that PBT reduces the amount of normal tissue exposed to irradiation compared with photon-based treatment, particularly at low- and mid-dose levels. Therefore, 3D-CRT and IMRT treatment planning was performed for the most recently treated case in the present study using the same CT images, in order to compare dose-volume histograms of the normal tissues, including the contralateral optic nerve, chiasm, brain and brain stem; the results are presented in Figs. 3 and 4. PBT improves the dose conformation compared with photon-based delivery methods due to the physical advantages of charged particle beams (6). Since PBT has the potential to reduce the irradiation dose to the organs at risk and to provide high dose irradiation to the tumor, tumor control and morbidity rates may be improved with this method (7-10).

However, the optimal dose required to control T4 SCC-ES tumors remains unknown (13). It is important to determine the appropriate total dose carefully while monitoring complications and escalating the total dose, which was achieved in the present study by gradually increasing the irradiation dose from 70 to 76 GyE. As a result, there was not a clear association between the irradiation dose and tumor control in the present study, mainly due to the small sample size and different tumor characteristics. A total of $4 / 7$ patients survived for $\geq 3$ years, and no lethal brain damage was observed irrespective of the irradiation dose. Therefore, patients with SCC-ES are currently being treated with PBT at a total dose of 76 GyE combined with concurrent chemotherapy at the University of Tsukuba 
Hospital. On the other hand, grade 3 optic nerve damage and cataracts developed in 1 and 2 patients, respectively, who received PBT. Previous studies have demonstrated the usefulness for spot-scanning proton therapy to significantly reduce the integral dose to head and neck critical structures $(19,20)$, and prospective studies are underway to determine if this reduced dose translates to an improved quality of life in patients.

In conclusion, 7 unresectable SCC-ES patients were treated with PBT with or without concurrent chemotherapy, and local tumor control was achieved in $3 / 7$ patients (43\%), while 4 patients survived for $\geq 3$ years. Furthermore, no lethal morbidity developed in the long-term survivors. As all recurrences developed locally, it appears that intensifying local treatment effects without increasing severe morbidities in critical organs may be important in order to improve treatment outcomes. PBT is therefore a potentially effective and curative RT method for unresectable SCC-ES.

\section{Acknowledgements}

A portion of the data obtained in the present study was presented at the 16th Asian Research Symposium in Rhinology held in Tokyo in 2013, and this study was supported by Grants-in-Aid for Scientific Research from the Ministry of Education, Culture, Sports, Science and Technology (no. 24591832) of Japan.

\section{References}

1. Blanco AI, Chao KS, Ozyigit G, et al: Carcinoma of paranasal sinuses: Long-term outcomes with radiotherapy. Int J Radiat Oncol Biol Phys 59: 51-58, 2004.

2. Jansen EP, Keus RB, Hilgers FJ, et al: Does the combination of radiotherapy and debulking surgery favor survival in paranasal sinus carcinoma? Int J Radiat Oncol Biol Phys 48: 27-35, 2000.

3. Dulguerov P, Jacobsen MS, Allal AS, Lehmann W and Calcaterra T: Nasal and paranasal sinus carcinoma: Are we making progress? a series of 220 patients and a systematic review. Cancer 92: 3012-3029, 2001

4. Jiang GL, Morrison WH, Garden AS, et al: Ethmoid sinus carcinomas: Natural history and treatment results. Radiother Oncol 49: 21-27, 1998.

5. Waldron JN, O'Sullivan B, Warde P, et al: Ethmoid sinus cancer: Twenty-nine cases managed with primary radiation therapy. Int $\mathbf{J}$ Radiat Oncol Biol Phys 41: 361-369, 1998.

6. Lomax AJ, Goitein M, Adams J, et al: Intensity modulation in radiotherapy: Photons versus protons in the paranasal sinus. Radiother Oncol 66: 11-18, 2003.
7. Hojo H,Zenda S, Akimoto T, et al: Impact of early radiological response evaluation on radiotherapeutic outcomes in the patients with nasal cavity and paranasal sinus malignancies. J Radiat Res 53: 704-709, 2012.

8. Truong MT, Kamat UR, Liebsch NJ, et al: Proton radiation therapy for primary sphenoid sinus malignancies: Treatment outcome and prognostic factors. Head Neck 31: 1297-1308, 2009.

9. Pommier P, Liebsch NJ, Deschler DG, et al: Proton beam radiation therapy for skull base adenoid cystic carcinoma. Arch Otolaryngol Head Neck Surg 132: 1242-1249, 2006.

10. Zenda S, Kohno R, Kawashima M, et al: Proton beam therapy for unresectable malignancies of the nasal cavity and paranasal sinuses. Int J Radiat Oncol Biol Phys 81: 1473-1478, 2011.

11. Suit $\mathrm{H}$ and Urie M: Proton beams in radiation therapy. J Nat Cancer Inst 84: 155-164, 1992.

12. Sobin LH, Wittekind C and Gospodarowicz M (eds): Nasal cavity and paranasal sinuses. In: TNM Classification of Malignant Tumors. 7th edition. Wiley-Blackwell, New York, NY, pp46-50, 2009.

13. Fukumitsu N, Okumura T, Mizumoto M, et al: Outcome of T4 (International Union Against Cancer Staging System, 7th edition) or recurrent nasal cavity and paranasal sinus carcinoma treated with proton beam. Int J Radiat Oncol Biol Phys 83: 704-711, 2012.

14. Therasse P, Arbuck SG, Eisenhauer EA, et al: New guidelines to evaluate the response to treatment in solid tumors. European organization for research and treatment of cancer, national cancer institute of the United States, national cancer institute of Canada. J Natl Cancer Inst 92: 205-216, 2000.

15. National Cancer Institute. Common Terminology Criteria for Adverse Events (CTCAE) v4.0 2010. http://evs.nci.nih.gov/ftp1/CTCAE/CTCAE_4.03_2010-06-14_ QuickReference_5'7.pdf. Accessed April 24, $201 \overline{4}$.

16. Hoppe BS, Nelson CJ, Gomez DR, et al: Unresectable carcinoma of the paranasal sinuses: Outcomes and toxicities. Int J Radiat Oncol Biol Phys 72: 763-769, 2008.

17. Padovani L, Pommier P, Clippe SS, et al: Three-dimensional conformal radiotherapy for paranasal sinus carcinoma: Clinical results for 25 patients. Int J Radiat Oncol Biol Phys 56: 169-176, 2003.

18. Mock U, Georg D, Bogner J, Auberger T and Pötter R: Treatment planning comparison of conventional, $3 \mathrm{~d}$ conformal and intensity-modulated photon (IMRT) and proton therapy for paranasal sinus carcinoma. Int J Radiat Oncol Biol Phys 58: 147-154, 2004.

19. Kandula S, Zhu X, Garden AS, et al: Spot-scanning beam proton therapy vs intensity-modulated radiation therapy for ipsilateral head and neck malignancies: A treatment planning comparison. Med Dosim 38: 390-394, 2013.

20. Flynn RT, Bowen SR, Bentzen SM, Rockwell Mackie T and Jeraj R: Intensity-modulated x-ray (IMXT) versus proton (IMPT) therapy for theragnostic hypoxia-based dose painting. Phys Med Biol 53: 4153-4167, 2008. 